\section{Bacterial life in space}

SIR - Your news item on the recent discussion meeting of the Royal Astronomical Society accurately reports the impasse between the two contending theories of interstellar grains. However, we would like to correct and clarify some technical points.

(1) H. Kroto's claim that there would be many ways of explaining the detailed profile of the 3.4 micrometre absorption feature was not substantiated by calculations based on any calibrated laboratory spectrum. No detailed fit on a satisfactorily large scale comparable with ours ${ }^{1}$ was shown at this meeting. Kroto's further claim that we had underestimated error bars on the astronomical observations was based on a hasty comparison between two graphs with different marked ordinate scales. When proper account is taken of these different ordinate scales there is no discrepancy. The statement that there is a wavelength shift between the peak absorption in bacteria near $\mathbf{3 . 4}$ micrometres and the astronomical observations was also incorrect. There is such a discrepancy when early astronomical data are used $^{2}$, but this was removed by later observations made independently of our laboratory measurements ${ }^{3,4}$. The latest data show a precise coincidence with the bacterial absorption near 3.4 micrometres and generally throughout the 2.9-3.9 micrometre waveband.

(2) The statements relating to the $2,800 \AA$ interstellar absorption due to amino acids (or lack of it) are worthy of comment. We did not raise this matter at the meeting as we felt it was sub judice because of objections that the IUE spectra were saturated near $2,800 \AA$. This may be so, but the internal evidence from the distribution of noise peaks did not indicate this, and when our student L.M. Karim obtained these spectra from Culham, he was not warned of a saturation problem. In any event the detection or otherwise of this band is peripheral to the identity of the grains.

(3) Neither side in the debate conceded any ground on the question of whether grains were biologic or not. However, both sides seem to have converged on the conclusion that grains must have a major organic component with at least 30 per cent of $\mathrm{C}$ in grains tied up in $\mathrm{CH}$ bonds. Ironically, precisely this situation was being strongly denied in your columns less than 5 years ago ${ }^{5,6}$. We might possibly be justified in claiming this agreement with our own earlier established position ${ }^{7-9}$ as a modest measure of success.

F. HOYLE

N. C. WICKRAMASINGHE

Department of Applied Mathematics

and Astronomy,

University College Cardiff,

Cardiff CFI $1 X L, U K$

1. Hoyle, F., Wickramasinghe, N.C., Al-Mufti, S., Olavesen, A.H. \& Wickramasinghe, D.T. Astrophys. Space Sci. $\mathbf{8 3}, 405$ (1982)
2. Hoyle, F., Wickramasinghe, N.C., Al-Mufti, S. \& Olavesen, A.H. Astrophys. Space Sci. 81, 489 (1982).

Allen, D.A. \& Wickramasinghe, D.T. Nature 294, 239 (1981) Jones, T.J., Hyland, A.R. \& Allen, D.A. Mon. Not. R. astr. Soc. 205, 187 (1983).

. D \& Williams, D.A. Nature 297, 40 (1979).

6. Whittet, D.C.B. Nature 281, 708 (1979)

Wickramasinghe, N.C. Nature 252, 462 (1974)

8. Hoyle, F. \& Wickramasinghe, N.C. Nature 264, 45 (1976); 268, 610 (1977).

9. Wickramasinghe, N.C., Hoyle, F., Brooks, J. \& Shaw, G. Nature 269, 674 (1977).

SIR - Philip Campbell's excellent report (Nature 17 November, p.218) of the Royal Astronomical Society Discussion Meeting “Are interstellar grains bacteria?" claimed that "at the end, none of the protagonists had conceded ground"'. Yet the article also reports that Hoyle and Wickramasinghe do not now claim that interstellar bacteria are alive. In his summary, Hoyle was forced into this position by the results of laboratory work by Greenberg at Leiden on the survival of bacteria subject to UV irradiation.

This claim by Hoyle is clearly the last of many evolutionary steps in the "bacteria" hypothesis. For, if the bacteria are no longer alive, they cannot restore themselves after chemical or radiative damage. Thus, within a few thousand years, they lose all their desirable optical properties, and cannot, therefore, supply the observed interstellar spectral features.

The Nature report did not mention the difficulties arising from interstellar depletion studies, as discussed at the meeting. Briefly, if the Sun gives the standard relative abundances of the elements in the interstellar medium, then the requirements of the bacteria model exceed the availability of both oxygen and phosphorus. Those attending the meeting were also impressed by photographs, presented by McDonnell, of recovered interplanetary dust in which silicate crystals were clearly visible. If siliceous material is responsible for the 10 micrometre feature observed in emission and absorption in the interstellar medium, then "bacteria" are again redundant, and in any case do not supply a good fit to the observed profile, as Butchart and Whittet have shown.

Department of Mathematics,

University of Manchester

Institute of Science and Technology, Manchester M60 1QD, UK

\section{Nobel Assembly}

SIR - In connection with your article "Nobel Prize to Barbara McClintock", Nature 13 October, p.575, I should like to point out that the Nobel Prize in Physiology or Medicine is awarded by the Nobel Assembly at the Karolinska Institute in Stockholm and not by the Swedish Academy.

\section{Secretary General,}

The Nobel Assembly,

Karolinska Institute,

S-104 01 Stockholm, Sweden

\section{Yellow raine}

SIR - The correspondence in Nature on the subject of "yellow rain" reminds me of an observation made by Charles Darwin. A "yellow rain" was noted more than a century ago in the Gardeners' Chronicle and Agricultural Gazette, no.29, 18 July 1863 , p.675. Paraphrased below are the contents of this paper and a letter by Darwin which was incorporated into the article. This information was gathered from Vol.II of The Collected Papers of Charles Darwin, edited by P. H. Barrett.

"Mr Charles Darwin forwarded a letter regarding the occurrence of a very slight shower lasting hardly more than a minute on the morning of July 2 about 10 o'clock. Emma Darwin gathered flowers shortly after the shower and noticed that the drops of water appeared yellowish and the white roses were all spotted and stained. Microscopic examination revealed brown spherical bodies $1 / 1,000$ of an inch in diameter and covered with short, conical transparent spines, and other smaller smooth colourless sacs about 4/7,000 of an inch in diameter. The water swarmed with elongated moving atoms only just visible. The petals on drying seemed to contain an impalpable matter the colour of rust of iron. Mr Darwin suggests that perhaps the Rev M.J. Berkeley might tell us what the larger spherical bodies are which fell this day by myriads from the sky carried by some distant whirlwind.

"Some days after the event another individual examined a leaf spotted with yellow dusty patches finding grains of Fir pollen and a few spores of Fungi along with spots from another unidentified plant but the principal constituent consisted of a slightly ferruginous apparently siliceous dust. This observer states that Fir pollen is often carried by the wind and deposited by rain on leaves. The pollen observed bore a strong resemblance to the grains of some Malvaceous plant but Malva sylvestris is the only species which could supply sufficient quantities of pollen to tinge the rain with a yellow tint; however, that species of pollen is smaller than the particles Darwin observed. The conclusion drawn was that it is quite astonishing what a multitude of bodies are carried about by the wind in the form of dust."

\section{Department of Biochemistry and}

BRAYDON C. GUILD

Molecular Biology,

Harvard University, Cambridge, Massachusetts 02138, USA

\section{Imperialist threat?}

SIR - I was disturbed when I first saw M.G. Audley-Charles's "Reconstruction of eastern Gondwanaland" (Nature 3 November, p.48). An engineering project of this magnitude, unprecedented in my knowledge, could only be financed and undertaken by a consortium of Western nations. Yet, the land masses involved are, for the most part, of the "Third World". Is this an appropriate use of resources and technology? And by what right do we disrupt other continents? Imagine my relief when I learned it was a theoretical reconstruction. JOHN T. DURKIN Harvard Medical School, Boston, Massachusetts 02115, USA 